\title{
Silicon Rich Oxide with controlled mean size of silicon nanocrystals by deposition in multilayers
}

\author{
E. Quiroga ${ }^{1 *}$, W. Bensch ${ }^{1}$, M. Aceves ${ }^{2}$, Z. Yu ${ }^{2}$, J. P. Savy ${ }^{3}$, M. Haeckel ${ }^{3}$, A. Lechner ${ }^{4}$ \\ ${ }^{1}$ Institute for Inorganic Chemistry of the University of Kiel. Germany \\ ${ }^{2}$ National Institute for Astrophysics, Optics and Electronics. Puebla, Mexico \\ ${ }^{3}$ Leibniz Institute for Marine Sciences. Kiel, Germany \\ ${ }^{4}$ University of Applied Sciences Regensburg. Germany \\ E-mail: equiroga@ieee.org
}

\begin{abstract}
The size of Si nanocrystals in Silicon Rich Oxide has been varied by depositing this material in multilayer arrays. They are possible candidates for one dimensional quantum devices. A study based on TEM, Raman and XRD measurements is presented.
\end{abstract}

Keywords — Silicon Rich Oxide, SRO, Si nanocrystals, size control, multilayer, Raman for size determination.

\section{INTRODUCTION}

Silicon nanocrystals (Si-nCs) as porous silicon or embedded in other materials have shown very interesting properties, useful in different fields. Among other properties, Si-nCs exhibit photoluminescence in the visible range, attributed to quantum confinement effects and to defects at the interfaces [1]. Additionally, some studies have been performed to use the $\mathrm{nCs}$ as charge storing centers in single buried layers [2,3] or superlattices of polysilicon [4].

Si-nCs can be created during the deposition of Silicon Rich Oxide (SRO). In this material, also called offstoichiometric silicon oxide, the Si-nCs grow by segregation of $\mathrm{Si}$ and $\mathrm{SiO}_{2}$ faces by thermal treatments [5]. An indicator of the Si content in the material is the parameter Ro, which is the ratio of the partial pressure of the precursor gases (for example, $\mathrm{N}_{2} \mathrm{O}$ and $\mathrm{SiH}_{4}$ ) when it is prepared by gas phase deposition methods as CVD (Chemical Vapor Deposition).

Si-nCs in SRO present a three dimensional size confinement, and are also isolated from other nCs by silicon oxides. This last property distinguishes them from those created by deposition of nanolayers of polysilicon, and is useful for single electron charging [6]. Nevertheless, the average crystallite size depends on several factors like temperature, pressure and the ratio Ro.

Here it is proposed a silicon particle size control by deposition of SRO in multilayers, varying just the deposition time. A low Ro value is used to obtain a material close to SIPOS (semi-insulating polysilicon). The multilayers are fabricated by LPCVD (Low Pressure Chemical Vapor Deposition), which is a simple and conventional method allowing the preparation of homogenous and relatively smooth films. TEM, Raman and XRD measurements were performed in order to characterize the samples.

\section{EXPERIMENTAL}

\section{A. Fabrication}

A SRO film with Ro $=1($ SRO1) and two multilayers (M1 and M2) composed of stacks of SRO1 and SRO with Ro $=50$ (SRO50) were fabricated by LPCVD at $725^{\circ} \mathrm{C}$. The used substrates were p-type Si (100) wafers with 30-50 $\mathrm{Ohm}-\mathrm{cm}$ resistivity and quartz substrates. The precursor gases were $\mathrm{SiH}_{4}$ and $\mathrm{N}_{2} \mathrm{O}$. The deposition of the multilayers was done varying manually the amount of precursor gases during the deposition. As a final step, all the samples were annealed for $3 \mathrm{~h}$ at $1100^{\circ} \mathrm{C}$ in vacuum.

The multilayer M1 consists of 6 layers of SRO50 alternating with 5 layers of SRO1. M2 is composed of 3 layers of SRO50 alternating with 2 SRO1 layers. The deposition times for every layer are listed in Table I.

\section{B. Characterization methods}

The microstructure of the samples was studied with TEM (Tecnai F30, $300 \mathrm{kV}$ ). XRD (X-ray Diffractometry) measurements were performed with a PANalytical X'PERTPRO diffractometer in reflection mode, using $\mathrm{Cu} \mathrm{K \alpha}$ radiation. Raman spectra were measured with a HORIBA Jobin Yvon LabRam HR, using $532 \mathrm{~nm}$ (frequency doubled Nd:YAG) laser excitation, a 100x Olympus objective with 0.9 numerical aperture, and an 1800 groove $/ \mathrm{mm}$ grating with a spectral resolution of about $1 \mathrm{~cm}^{-1}$. Approximately $10 \mathrm{~mW}$ laser light was focused on each sample in order to avoid artifacts in the measurements due to heating. The measurements were performed in dark to avoid any interference with light.

TABLE I

DEPOSITION TIMES FOR THE SAMPLES

\begin{tabular}{ccccc} 
Sample & $\begin{array}{c}\text { Number } \\
\text { of layers } \\
\text { of } \\
\text { SRO50 }\end{array}$ & $\begin{array}{c}\text { SRO50 } \\
\text { dep. time } \\
\text { (min) }\end{array}$ & $\begin{array}{l}\text { Number } \\
\text { of layers } \\
\text { of SRO1 }\end{array}$ & $\begin{array}{c}\text { SRO1 } \\
\text { dep. time } \\
\text { (min) }\end{array}$ \\
\hline SRO1 & - & - & 1 & 45 \\
M1 & 6 & 14 & 5 & 5 \\
M2 & 3 & 20 & 2 & 10 \\
\hline
\end{tabular}




\section{RESULTS AND DISCUSSION}

\section{A. TEM observations}

SRO1 is a material with characteristics similar to SIPOS (semi-insulating polysilicon) due to its high Si content [7]. $\mathrm{Si}$ agglomerates nucleate during the deposition process. After high temperature annealing, well defined $\mathrm{Si}$ nanocrystals (Si-nCs) are obtained from the $\mathrm{Si}$ agglomerates, as can be observed in Fig. 1. According to the observations on the samples, the nCs are homogenously distributed and their size varies between 10 and $35 \mathrm{~nm}$. The $\mathrm{nCs}$ present a random orientation.

Multilayer M2 is well defined (Fig. 2) evidencing the deposition of all the layers. The SRO1 layers in M2 have a thickness of around $22 \mathrm{~nm}$. On the other hand, the layers in M1 cannot be easily identified by TEM, probably due to the high roughness of the SRO1 layers and due to the very thin SRO50 layers. Nevertheless, the SRO1 layers in M1 should be separated by layers of SRO50, even when they are not smooth, since large bright regions of amorphous material (SRO50) are identified by TEM.

\section{B. Raman measurements}

Fig. 3 shows the Raman spectra of the samples deposited on quartz substrate. The spectrum of bulk silicon is included for comparison. The Raman peak at $511 \mathrm{~cm}^{-1}$ is related to that at $520 \mathrm{~cm}^{-1}$ of crystalline $\mathrm{Si}$ (c-Si). This peak $\left(\Gamma_{25}{ }_{25}\right)$ is the optical phonon mode in the center of the Brillouin zone [8], but it is slightly shifted due to quantum confinement effects [9]. The peak around $300 \mathrm{~cm}^{-1}$ is due to the two phonon 2TA mode of $\mathrm{c}-\mathrm{Si}$, and the intensity is enhanced by LA phonons of an amorphous $\mathrm{Si}$ (a-Si) phase [8]. Actually, the peak around $480 \mathrm{~cm}^{-1}$ occurring as a shoulder corresponds to LO phonons in a-Si [8, 9]. These results suggest that the $\mathrm{Si}$ agglomerates in $\mathrm{SRO} 1$ are not totally crystallized even after thermal treatments.

The position of the $\Gamma_{25}^{\prime}$ line is approximately the same in all the samples, contrary to the expected difference due to different nanocrystal sizes from sample to sample. The position and the width of the $\Gamma_{25}^{\prime}$ peak are used by different groups to estimate the $\mathrm{Si}$ crystallite size [9 - 12]. But one should keep in mind that both position and width may be strongly affected by stress, roughness, or missfocusing [9]. Therefore, other parameters should be used for a qualitative estimation of the crystalite size.

A broadening of the $\Gamma^{\prime}{ }_{25}$ peak on the low wavenumber range being visible as a shoulder is caused by a distribution of crystallite sizes [10]. Hence, the shape of the peak could give an idea of the average crystallite size of $\mathrm{Si}$. The ratio of the left width at half maximum (LWHM) to the right width at half maximum (RWHM) is the asymmetry coefficient $C_{a}$. In this work $\mathrm{C}_{\mathrm{a}}$ is considered as an indication of the shape of the $\Gamma^{\prime}{ }_{25}$ peak. Since $C_{a}$ is a relative measure of the shape of the Raman peak, it is independent of its absolute width, height or Raman shift, and consequently insensitive against external factors. The values of $\mathrm{C}_{\mathrm{a}}$ for the different samples are shown in Table II.

It has been reported that when the $\mathrm{Si}$ nanocrystal size decreases, the $\Gamma_{25}^{\prime}$ peak broadens [9]. The fact that $\mathrm{C}_{\mathrm{a}}$ increases when the deposition time of SRO1 decreases may indicate that the crystal size decreases, as is corroborated in the XRD section.

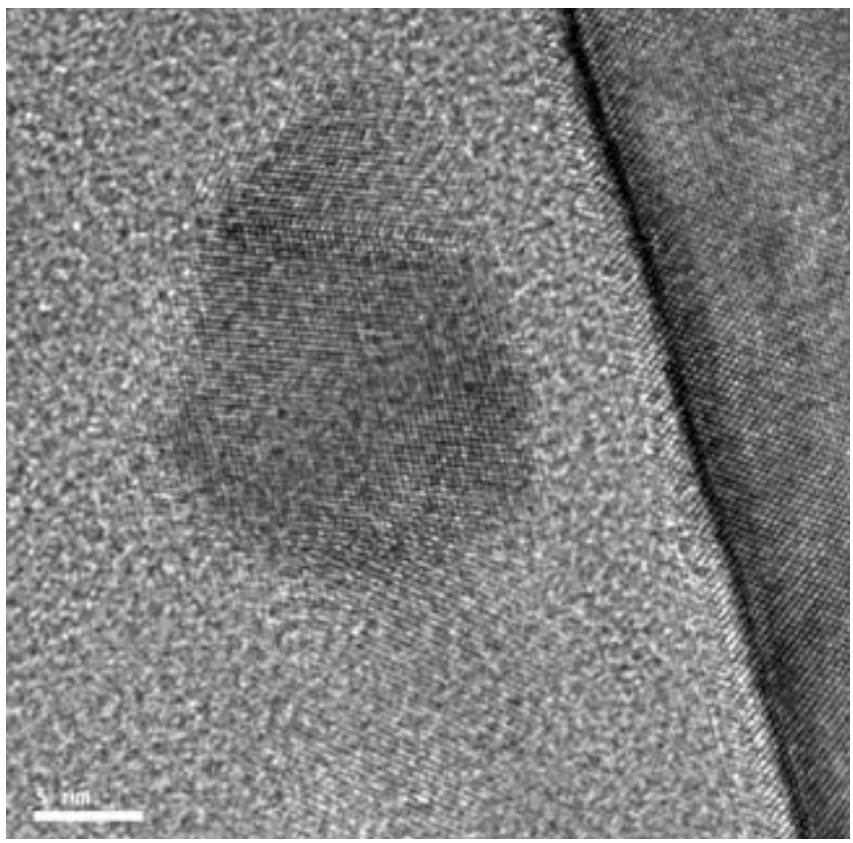

Fig. 1. Typical TEM micrograph of SRO1 in M1. A nanocrystal with diameter of $\sim 20 \mathrm{~nm}$ is clearly observed.

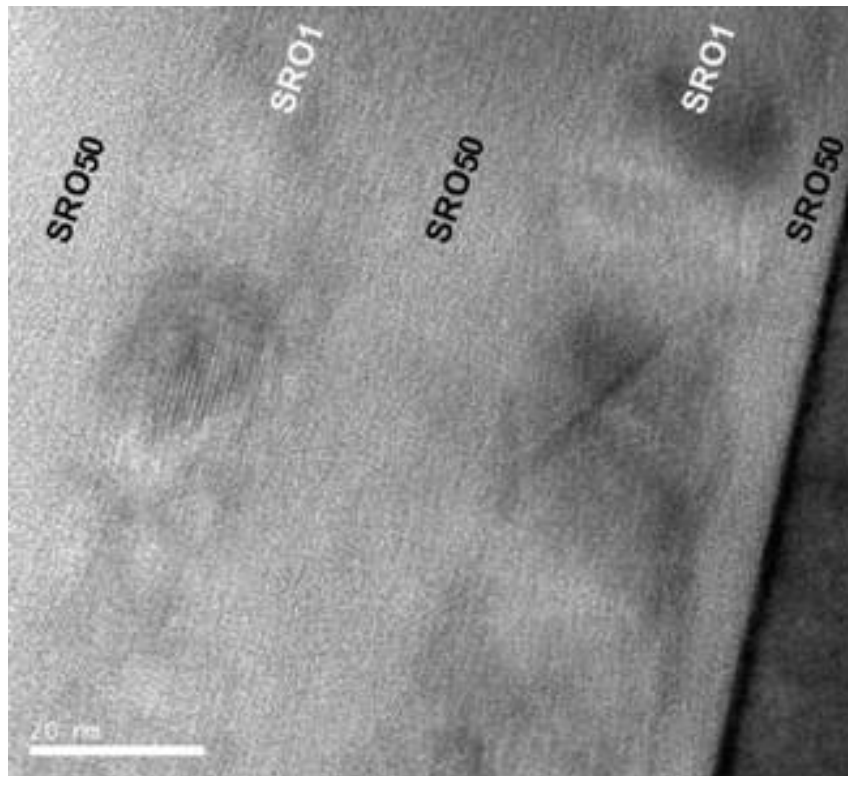

Fig. 2. TEM of M2. The dark zones are layers of SRO1, and the bright ones are layers of SRO50. 


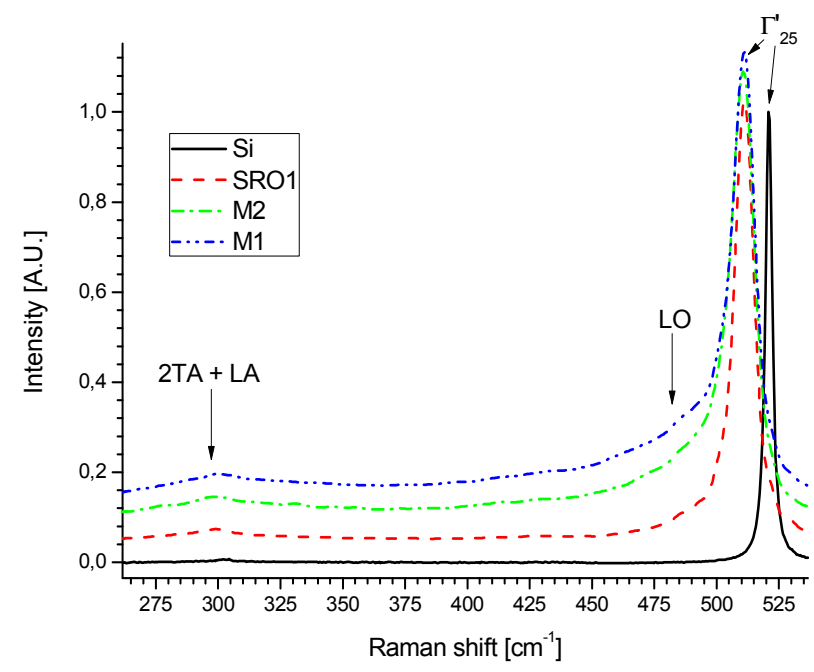

Fig. 3. Raman Spectra of the samples. The spectrum of bulk Si is included for comparison. Three lines related to Si are identified: 2TA and LA (300 $\left.\mathrm{cm}^{-1}\right)$, $\mathrm{LO}\left(480 \mathrm{~cm}^{-1}\right)$ and $\Gamma_{25}\left(520 \mathrm{~cm}^{-1}\right.$ in bulk Si, and $511 \mathrm{~cm}^{-1}$ in the rest of the samples).

TABLE II

ASYMMETRY OF THE $\Gamma{ }_{25}$ LINE IN THE STUDIED SAMPLES

\begin{tabular}{ccc} 
Sample & $\begin{array}{c}\text { SRO1 } \\
\text { dep. time } \\
\text { (min) }\end{array}$ & $\mathrm{C}_{\mathrm{a}}$ \\
\hline $\boldsymbol{S R O 1}$ & 45 & 1.136 \\
$\boldsymbol{M} \mathbf{2}$ & 10 & 1.242 \\
$\boldsymbol{M 1}$ & 5 & 1.345 \\
\hline
\end{tabular}

\section{XRD measurements}

Samples deposited on quartz were used to detect the reflections of Si-nCs, The measurements were performed with long collection times to improve the statistics, but even so, due to the low thickness of the layers the intensity of the reflections is low. Nevertheless, the patterns of all samples evidently present the characteristic reflections of $\mathrm{c}-\mathrm{Si}$ (111) and (220) at $28^{\circ}$ and $47.3^{\circ} 2 \theta$ respectively [13] (see Fig. 4).

Assuming that the samples present a random orientation of nCs, the most intense (111) reflection is taken to calculate the crystallite size. To estimate the size of the coherent scattering domains the Scherrer equation [14] was used:

$$
B=\frac{K \lambda}{L \cos \left(\frac{\chi}{2}\right)}
$$

where $\mathrm{B}$ is the FWHM of the reflection in radians, $\mathrm{K}$ is a constant for which a value of 0.9 is widely accepted assuming spherical particles; $\lambda$ is the wavelength of incident $\mathrm{X}$-rays $(0.1504 \mathrm{~nm}) ; \chi$ is the center position of the peak, and $\mathrm{L}$ is the crystallite length. The parameters and results of this calculation are summarized in Table III.

According to the data compiled in Table III, the Si nanocrystal size is smaller for shorter deposition times of
SRO1, as was also evidenced by the Raman measurements. Fig. 5 shows the relation of the crystallite size with the parameter $\mathrm{C}_{\mathrm{a}}$. In the presented range of nanocrystal sizes the relation seems to be parabolic, but more experiments are needed to probe this tendency and to be able to use this analysis in the estimation of particle sizes.

According to these results it can be concluded that the average size of the Si-nCs in SRO1 $(\sim 30 \mathrm{~nm}$ in thick single films) is limited by the layer thickness. In this way, the size of the nCs can be controlled by tuning the layer thickness.

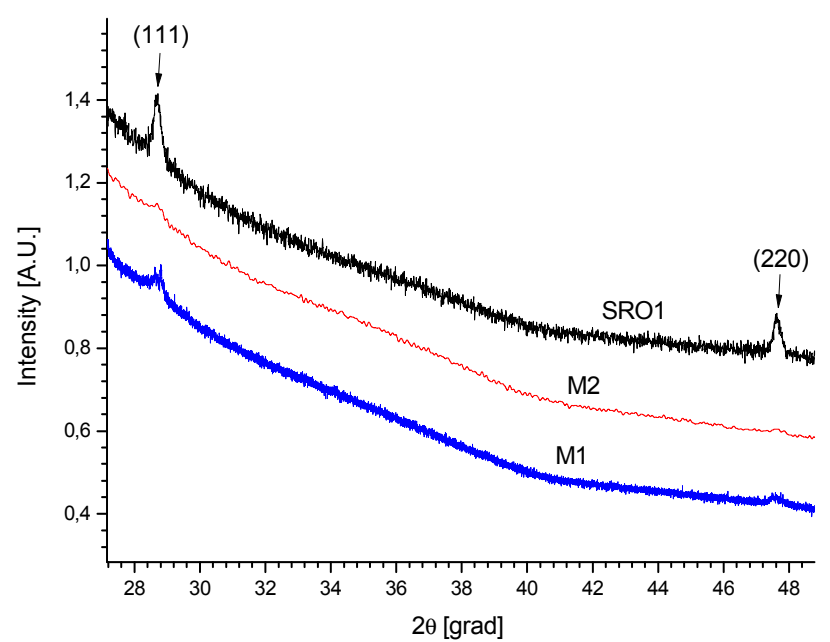

Fig. 4. XRD difractogram of the studied samples. The characteristic reflections of crystalline $\mathrm{Si}$ (111) and (220) are identified.

TABLE III NANOCRYSTAL SIZE

\begin{tabular}{cccc} 
Sample & $2 \theta[\mathrm{grad}]$ & $\begin{array}{c}\text { FWHM } \\
{[\mathrm{grad}]}\end{array}$ & $\begin{array}{c}\text { Size } \\
{[\mathrm{nm}]}\end{array}$ \\
\hline SRO1 & 28.69 & 0.275 & 29.8 \\
M2 & 28.71 & 0.337 & 24.3 \\
M1 & 28.73 & 0.378 & 21.7 \\
\hline
\end{tabular}

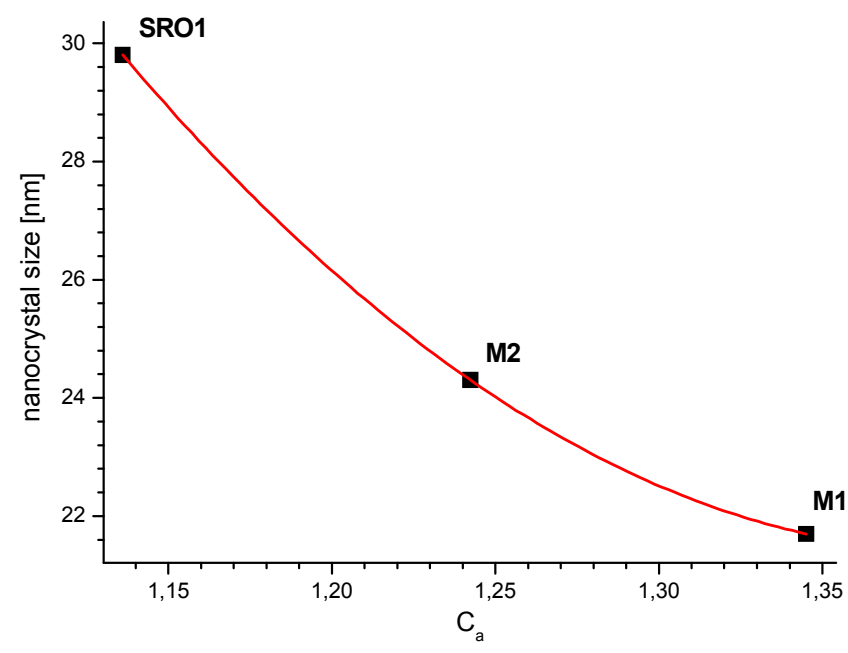

Fig. 5. Relation of the nanocrystal sizes with the parameter $\mathrm{C}_{\mathrm{a}}$. 
Additionally, the nCs may tend to be elliptical, since the SRO1 layer thickness in M2 is $22 \mathrm{~nm}$ (from TEM) and the average nanocrystal size is $24.3 \mathrm{~nm}$. But this small difference indicates that the shape is almost spherical.

\section{CONCLUSION}

The size of $\mathrm{Si}$ nanocrystals (Si-nCs) in $\mathrm{SRO} 1$ in multilayer arrays depends on the layer deposition time. This is evidenced by studying the asymmetry of the Raman line $\Gamma_{25}^{\prime}$ of Si and by XRD. Evaluation of the XRD data allowed a semi-quantitative estimation of the crystallite sizes in different films. Furthermore, the relation of the crystallite size with the asymmetry coefficient $C_{a}$ seems to be parabolic. Nevertheless, further studies are needed for establishing the analysis of the asymmetry of Raman lines as a tool for the determination of the size of Si-nCs.

The dependency of the Si nanocrystal size on the layer deposition time can be interpreted as a size control, because in one dimension the growth of the $\mathrm{nCs}$ is limited by the layer thickness. The $\mathrm{nCs}$ are isolated by $\mathrm{SiO}_{2}$ allowing their use in many applications, e.g. as one dimensional quantum devices.

\section{ACKNOWLEDGMENT}

The authors appreciate the support from CONACyT and DAAD. Jürgen Kolouch is thanked for his technical collaboration.

\section{REFERENCES}

[1] D. Berman, M. Aceves, A. Gallegos, A. Morales, L. R. Berriel, J. Carrillo, F. Flores, C. Falcony, C. Domínguez, A. Llobera, M. Riera, and J. Pedraza "Silicon excess and thermal annealing effects on the photoluminescence of $\mathrm{SiO}_{2}$ and silicon rich oxide super enriched with silicon implantation," phys. stat. sol. (c) 1, No. S1, S83- S87 (2004).

[2] D. N. Kouvastos, V. Ioanou-Sougleridis, A. G. Nassiopoulou, "Charging effects in silicon nanocrystals within $\mathrm{SiO}_{2}$ layers, fabricated by chemical vapor deposition, oxidation and annealing," Appl. Phys. Lett. 82, No. 3, pp. 397-399, (2003).

[3] V. Ioanou-Sougleridis, A. G. Nassiopoulou, "Investigation of charging phenomena in silicon nanocrystal metal-oxidesemiconductor capacitors using ramp current-voltage measurements," J. Appl. Phys. 94, No. 6, pp. 4084-4087, (2003).

[4] E. Quiroga, Z. Yu, M. Aceves, "Charging/discharging effects in nc$\mathrm{Si} / \mathrm{SiO}_{2}$ superlattice prepared by LPCVD" in: Proceedings 2nd International Conference on Electrical and Electronics Engineering (ICEEE) and XI Conference on Electrical Engineering (CIE 2005). Mexico City, Mexico, 2005, pp. 219-222, (IEEE, 2005).

[5] B. Fazio, M. Vulpio, C. Gerardi, Y. Liao, I. Crupi, S. Lombardo, S. Trusso, F. Neri, "Residual crystalline silicon phase in Silicon-RichOxide films subjected to high temperature annealing," Journal of the electrochemical society 149, No. 7, pp. G376-G378 (2002).

[6] Z. Yu, M. Aceves, J. Carrillo, F. Flores, "Single electron charging in Si nanocrystals embedded in silicon-rich oxide," Nanotechnology, 14, pp. 959-964 (2003).
[7] E. Quiroga, W. Bensch, Z. Yu, M. Aceves, R. A. De Souza, M Martin, V. Zaporojtchenko, F. Faupel, "Structural characteristics of a multilayer of Silicon Rich Oxide (SRO) with high Si content prepared by LPCVD," phys. stat. sol. (a). 206, No. 2, pp. 263-269 (2009).

[8] Y. Ma, X. Liao, G. Kong, J Chu, "Raman scattering of nanocrystalline silicon embedded in $\mathrm{SiO}_{2}$," Science in China (Series A) 43, No. 4, pp. 414-420 (2000)

[9] Ch. Ossadnik, S Vepřek, I. Gregora, "Raman Applicability of Raman scattering for the characterization of nanocrystalline silicon," Thin Solid Films 337, pp. 148-151 (1999).

[10] S. V. Gă̌sler, O. I. Semenova, R. G. Sharafutdinov, B. A. Kolesov, "Analysis of raman spectra of amorphous-nanocrystalline silicon films," Physics of the Solid State 46, No. 8, pp. 1528-1532 (2004).

[11] H. S. Mavi, A. K. Shukla, R. Kumar, S. Rath, B. Joshi, S. S. Islam, "Quantum confinement effects in silicon nanocrystals produced by laser-induced etching and cw laser annealing," Physics Semicond. Sci. Technol. 21, pp. 1627-1632 (2006).

[12] B. Li, D. Yu, S. L. Zhang, "Raman spectral study of silicon nanowires," Physics Semicond. Sci. Technol. 21, pp. 1627-1632 (2006).

[13] A. Le Donne, S. Binetti, G. Isella, B. Pichaud, M. Texier, M. Acciarri, S. Pizzini, "Structural characterization of nc-Si films grown by lowenergy PECVD on different substrates," Applied Surface Science 254, pp. 2804-2808 (2008).

[14] A. L. Patterson, "The Scherrer formula for x-ray particle size determination," Physical Review 56, pp. 978-982 (1939). 